\title{
Die Röntgen Akademie für Führungskräfte in der Radiologie stellt sich vor
}

Bisher wurde in der medizinischen Ausbildung das Thema Mitarbeiterführung kaum behandelt. In einer Zeit komplexer Anforderungen und Arbeitsverdichtung spielen aber zunehmend professionelle Führungskompetenzen eine entscheidende Rolle für den Erfolg von Kliniken und Arztpraxen. Das Motivieren, das verantwortliche, wirksame Führen von Mitarbeitern, sowie die Lösung von Konflikten am Arbeitsplatz werden zunehmend wichtiger. Veränderungsprozesse in Einrichtungen des Gesundheitswesens sind heute Alltagsgeschäft.

Die innerhalb der Deutschen Röntgengesellschaft neu gegründete Röntgen Akademie für Führungskräfte bietet erstmalig hochprofessionelle Seminare und Workshops speziell für angehende Führungskräfte und junge Chefärzte oder niedergelassene Ärzte in der Radiologie an.

Für diese Fortbildungsveranstaltungen konnten wir Herrn Jens Hollmann gewinnen. Er ist Inhaber des Unternehmens medplus-kompetenz, Lehrbeauftragter an der Donau-Universität Krems, Coach und Berater für Ärzte und Klinikleitungen und bereits manch einem durch seinen

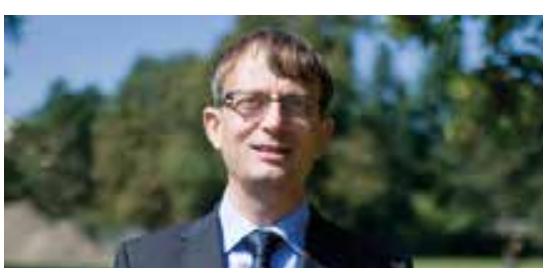

Jens

Hollman

Vortrag beim Chefarztforum (CAFRAD) bekannt. Seine Themenfelder umfassen den Bereich „Wirksames Führen“ (Motivation, Konflikt-Management, ChangeManagement, Teamentwicklung), sowie das Thema "Selbstmanagement für ärztliche Leistungsträger". Er ist außerdem Autor zahlreicher Publikationen zu genannten Themen.

\section{Eindrücke vom 1. Führungskräfte- seminar für Radiologen}

Das 1. Seminar mit dem Titel „Die erste Zeit als Chefarzt - Teamführung, Mitarbeitergespräche, Konfliktmanagement" fand statt am 10. und 11. Juli 2015 in Innsbruck, Österreich statt.

Für die Teilnehmer begann das Programm im Innsbrucker Kongresspark Igls bei strahlendem Sonnenschein mitten in den 


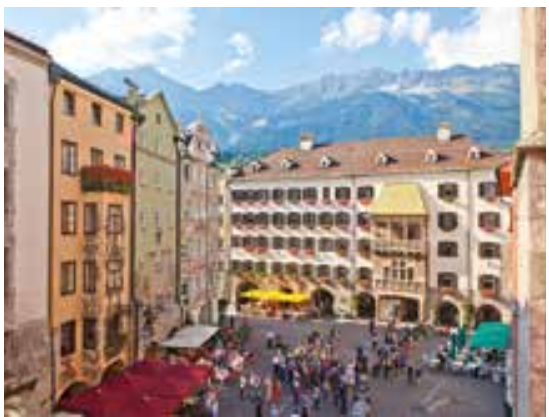

Tiroler Alpen, umgeben von einer einzigartigen Naturkulisse.

Von Anfang an zeigte sich, wie kompetent der Referent Jens Hollamnn (medplus) ist, wie sehr die gute Organisation dieses Workshops und der Veranstaltungsort zum Gelingen dieser Veranstaltung beitrugen. Das Verhältnis von Theorie und praxisbezogenen Beispielen war sehr ausgewogen und wurde durch interessante Gespräche der Teilnehmer untereinander während der Pausen bereichert. Jens Hollmann konnte sehr schnell das Vertrauen der Teilnehmer gewinnen. Er begeisterte nicht nur durch seine Kompetenz, sondern auch durch seine Erfahrungsberichte aus der Praxis und die Fähigkeit auf die Teilnehmer einzugehen. Nach kurzer Zeit wurde anhand konkreter Beispiele der Teilnehmer diskutiert.

Am Abend ging es dann von Innsbruck aus mit der Stand- und Luftseilbahn (Hungerburg- und Seegrubenbahn) in Richtung Restaurant Seegrube, das auf knapp $2000 \mathrm{~m}$ Höhe in der Tiroler Alpenlandschaft liegt und einen unvergesslichen Ausblick ins Tal und auf Innsbruck bietet. Hier konnten die Erfahrungen ausgetauscht und Erlerntes in lockerer Atmosphäre diskutiert werden.

Der 2. Seminartag fand im Hotel aDLERS statt. In einem Seminarraum in der 12 . Etage mit einem Blick über die Dächer von
Innsbruck und die einrahmende Berglandschaft fokussierte Jens Hollmann den Schwerpunkt auf das Thema Umgang mit Konflikten. Was bedeutet Konfliktfelderkundung und wie löse ich Konflikte im Klinikalltag? Diese Fragen wurden eingehend behandelt und boten genug Diskussionsstoff für die abschließende Gesprächsrunde und den Erfahrungsaustausch mit erfahrenen Chefärzten, wie Prof. Dr. med. Gabriele Krombach, PD Dr. med. Wolfgang Krings und Prof. Dr. med. Dierk Vorwerk.

Zum Abschluss des Seminars bot sich für jeden Teilnehmer, die Möglichkeit sein eigenes Konfliktverhalten zu analysieren.

Die 2 Tage waren ein voller Erfolg und das 1. Führungskräftetraining für Radiologen in dieser Art, gleichzeitig aber auch ein interessanter Erfahrungsaustausch mit gleichgestellten Kollegen, aber auch „alten Hasen“.

\section{Veranstaltungsreihe für Oberärzte auf dem Weg in eine Führungs- position $\nabla$}

Am 13. und 14. November wird in Berlin das 1. Seminar (Basiskurs) für Oberärzte stattfinden. In diesem Basiskurs und 5 weiteren Modulen werden die Teilnehmer auf das Bewerbungsverfahren und ihre zukünftigen Aufgaben als Chefarzt vorbereitet.

Der Basiskurs beinhaltet zunächst folgende Themen: Selbsterkenntnis, Selbstführung, Assessmentcenter, Karriereauswahlverfahren (vorgestellt durch einen Personalberater), evidenzbasierte Führungsforschung, erfolgreiche Kommunikation.
In den einzelnen Modulen (Selbstmanagement, Konfliktmanagement, Team und Motivation, Changemangement, Ökonomische Aspekte) werden die erforderlichen Kompetenzen und Methoden für das Management im Klinikalltag vermittelt.

Die einzelnen Module sind zertifiziert von der Röntgen Akademie für Führungskräfte in der Radiologie. Sie erhalten ein Zertifikat nach erfolgreicher Teilnahme am Basiskurs und 3 weiteren Modulen.

Geplant ist eine Kooperation mit der Donau Universität Krems für den Abschluss Management im Gesundheitswesen/Healthcare Management - MBA (Anrechenbarkeit für den Bereich Führung).

\section{Wie geht es weiter? \\ $\nabla$}

Weitere Seminare auch für Ärzte, die bereits in leitender Funktion in Klinik oder Niederlassung tätig sind, werden folgen. Das nächste ist geplant für den Herbst 2016, der nächste Basiskurs für Oberärzte wird bereits im Frühjahr 2016 stattfinden.

Denn ... um zeitgemäß und in die Zukunft zu führen, ist es erforderlich über eine entsprechende Bandbreite an Modellen und Führungsansätzen zu verfügen und diese überzeugend einsetzen zu können. Wir helfen Ihnen dabei!

Informieren Sie sich am besten gleich über www.drgakademie.de - Röntgen Akademie

\section{Kontakt:}

Röntgen Akademie für Führungskräfte in der Radiologie

Deutsche Röntgengesellschaft e.V.

Frau Sabine Schlender

Tel.: 030-91607011

Email: schlender@drg.de

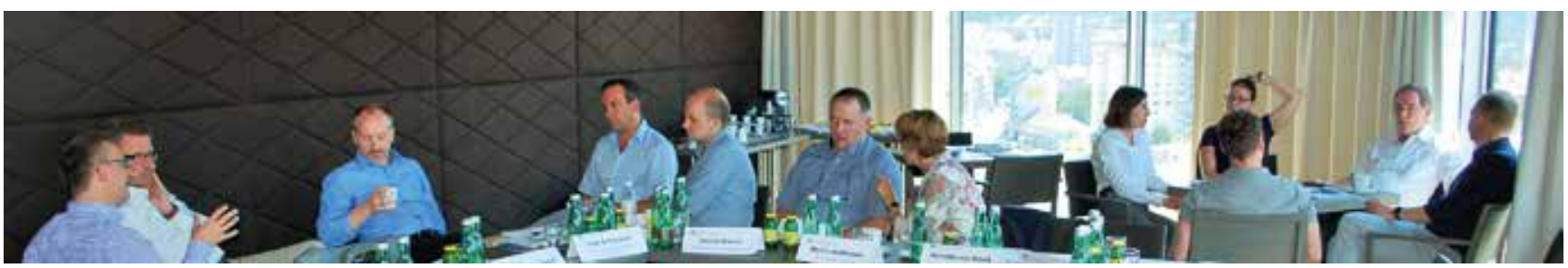

